\title{
A Linear Program Formulation for the Segmentation of Ciona Membrane Volumes
}

\author{
Diana L. Delibaltov ${ }^{1}$, Pratim Ghosh ${ }^{1}$, Volkan Rodoplu ${ }^{1}$, Michael Veeman ${ }^{2,3}$, \\ William Smith ${ }^{2}$, and B.S. Manjunath ${ }^{1, \star}$ \\ ${ }^{1}$ Department of Electrical and Computer Engineering \\ 2 Department of Molecular, Cellular and Developmental Biology \\ ${ }^{2,1}$ University of California, Santa Barbara, CA - 93106 \\ ${ }^{3}$ Division of Biology, Kansas State University, Manhattan, KS - 66506
}

\begin{abstract}
We address the problem of cell segmentation in confocal microscopy membrane volumes of the ascidian Ciona used in the study of morphogenesis. The primary challenges are non-uniform and patchy membrane staining and faint spurious boundaries from other organelles (e.g. nuclei). Traditional segmentation methods incorrectly attach to faint boundaries producing spurious edges. To address this problem, we propose a linear optimization framework for the joint correction of multiple over-segmentations obtained from different methods. The main idea motivating this approach is that multiple over-segmentations, resulting from a pool of methods with various parameters, are likely to agree on the correct segment boundaries, while spurious boundaries are methodor parameter-dependent. The challenge is to make an optimized decision on selecting the correct boundaries while discarding the spurious ones. The proposed unsupervised method achieves better performance than state of the art methods for cell segmentation from membrane images.
\end{abstract}

Keywords: Cell segmentation, Linear program, Joint segmentation.

\section{Introduction}

Embryonic morphogenesis involves the emergence of shape at the cell, tissue, organ and organismal levels. A quantitative, systems-level understanding of this process will require a set of robust methods for segmentation and cell specific measurements (volume, shape analysis, etc). Ascidians are invertebrate chordates with particularly small, simple embryos. The major tissues in the ascidian are illustrated in Fig. 1(a). The images used here are confocal microscopy volumes of Ciona embryos where the cell peripheries have been stained [10. 1]

This work addresses the problem of cell segmentation, which is necessary to quantify biologically important parameters of the cell (size, shape, etc) 3]. This

\footnotetext{
* This work was supported by NIH HD059217 and NSF III-0808772.

1 The Ciona embryos were fixed, stained with Bodipy-FL phallicidin to label the cortical actin cytoskeleton, cleared in Murray's Clear (BABB), and imaged on an Olympus FV1000 LSCM using a 40x 1.3NA oil immersion objective.
} 


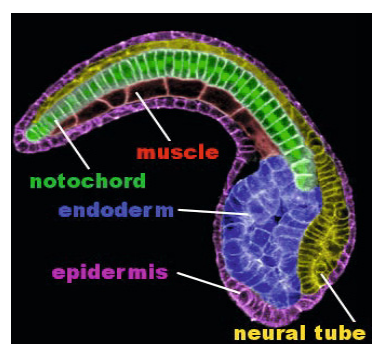

(a)

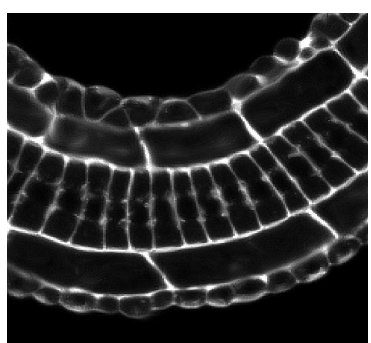

(b)

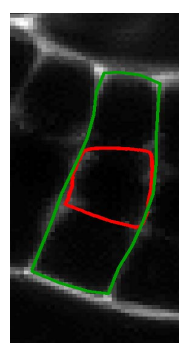

(c)

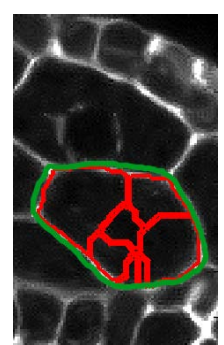

(d)

Fig. 1. Best viewed in color. (a) The major tissues of the ascidian tadpole. 9] (b) Detail of a confocal section. Notice the faint nuclei boundaries in the string of notochord cells. (c) Subjective surface 13 segmentation method attached to the faint nucleus boundary (red contour). (d) Watershed method [6] (red contour) initialized at local minima incorrectly fragments the cell. The correct segmentations are marked in green.

is a challenging dataset due to varying cortical intensity and faint staining of other organelles. As seen in Fig. 1(c) and 1(d), segmentation methods incorrectly attach to the faint nucleus boundaries captured by the staining. Furthermore, state of the art segmentation methods developed for confocal microscopy membrane volumes, such as [13, require the initialization with a seed point inside each cell of interest, as well as manually cropping the volume around each cell. For high-throughput analysis, it is preferable to have minimal or no human interaction.

Towards this, we tackle the task of 3-D segmentation of the Ciona volumes by simultaneously correcting multiple over-segmentations in a principled manner. We start out with the results of multiple segmentation methods resulting from a pool of methods, referred to as the bag of methods. The methods could differ in their segmentation scheme or in the choice of parameters for a single algorithm. We assume that all of these are tuned for over-segmentation, thus resulting in super-pixels, and containing more boundaries than necessary. Tuning a method for over-segmentation is a much easier task than searching for the narrow range of parameters which result in the desired boundaries. Furthermore, over-segmentations can be produced efficiently and in parallel, with a method such as [6], which is extremely suitable for edge data. The intuition is that the boundaries of interest are present in all the over-segmentations, along with many other spurious edges which are method- or parameter-dependent. We introduce a linear program framework for simultaneously correcting these over-segmented results in order to achieve consensus among the detected boundaries.

The problem of fusing multiple segmentations has caught interest in recent years in the computer vision community. Vitaladevuni et al., in [14, address the problem of jointly clustering two over-segmentations modelled as a quadratic semi-assignment program, which is relaxed to a linear program. Unlike this work, our method handles multiple over-segmentations and has fewer triangular inequalities. Warfield et. el [1] also combine a set near perfect segmentations 
from trained raters into one consensus segmentation. The method we propose solves a more challenging problem where the over-segmented inputs are far from correct. The authors of [8] propose a segmentation algorithm which learns a combination of weak segmenters and builds a strong one. The works of [12] and 7] propose label fusion methods where expert-segmented training images are registered to the target image. Unlike these methods, the proposed algorithm achieves a consensus in an unsupervised manner.

We propose a novel method which consists of a linear program optimization framework that simultaneously corrects multiple over-segmentations, such that the agreement between them is maximized. As a result of the convex formulation we can compute a globally optimal solution. The method is generalizable to twoor three-dimensional data. We present results on a $3-\mathrm{D}$ confocal microscopy volume of the membrane stained ascidian Ciona. Our method achieves better performance than state of the art segmentation methods for this type of data.

\section{Joint Correction of Multiple Over-Segmentations}

We assume that $N$ over-segmentations of the image $\mathcal{I}$ are available. These oversegmentations, denoted as $S_{1}-S_{N}$ can differ in their methodology or in the parameters of a single algorithm. Every label-map $S_{p}$ has a total of $N_{p}$ segments. We consider correcting these $N$ over-segmentations by merging segments with similar characteristics within each label map, while simultaneously obtaining the maximum agreement across the $N$ corrections (Fig. 2(f). The dissimilarity between neighboring segments within each label-map is characterized by a cost of merging. The agreement between two overlapping segments across two labelmaps is characterized by a reward for connecting segments across two consecutive over-segmentations. We formulate the problem as a binary integer program, which minimizes the total cost of merging segments within each label map, while maximizing the total reward for agreement across the segmentations. The binary integer program is further relaxed to a linear program.

The spatial relationship between the super-pixels in each segmentations is modelled as nodes in a graph. The initial over-segmentations are considered in arbitrary order, and every pair of consecutive over-segmentations are connected in the graph as shown in Fig. 2(f).

\subsection{Binary Integer Program Formulation}

We introduce the parameter $C^{p}$, an $N_{p} \times N_{p}$ dimensional matrix whose entries $C_{i j}^{p}$ represent the penalty (cost) for merging segments $i$ and $j$ within label-map $S_{p}$. The connectivity parameter $E^{p, p}$ is an $N_{p} \times N_{p}$ binary matrix whose entries $E_{i j}^{p, p}$ are 1 if segments $i$ and $j$ both from label-map $S_{p}$ are in contact by at least one pixel, and 0 otherwise.

We take the $N$ segmentations in arbitrary order and consider every two consecutive label-maps. The connectivity parameter across two consecutive label-maps $E^{p, p+1}$, is defined as $N_{p} \times N_{p+1}$ matrix, where $E_{i j}^{p, p+1}$ indicates if segment $i$ 


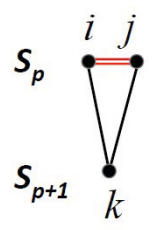

(a)

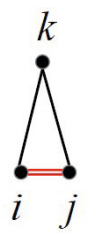

(b)

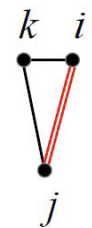

(c)

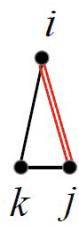

(d)

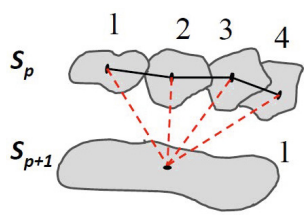

(e)

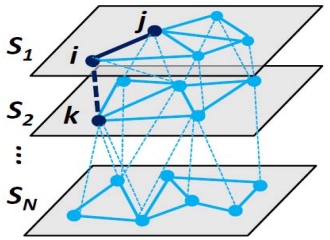

(f)

Fig. 2. (a)-(d) Given the black single line connections, the red double line connections result from transitivity constraints similar to Eq. (6). (e): Transitivity constraints are only active for neighboring segments, otherwise the LP is infeasible. This diagram shows an example where the transitivity and the connectivity constraints cannot both be satisfied. (f) Graph representation of the multiple over-segmentations. The solid dark line represents $m_{i, j}^{1,1}$, while the dotted dark line represents $m_{i, k}^{1,2}$.

from label-map $S_{p}$ and segment $j$ from label-map $S_{p+1}$ share at least one pixel. Consequently, the reward parameter, $R^{p, p+1}$, is represented by a $N_{p} \times N_{p+1}$ matrix, whose entries $R_{i j}^{p, p+1}$ quantify the agreement between segment $i \in S_{p}$ and $j \in S_{p+1}$.

Next, we introduce the decision variables. Variable $m^{p, p}$ is a $N_{p} \times N_{p}$ binary matrix whose entries $m_{i j}^{p, p}$ are non-zero if the two segments $i$ and $j$ from labelmap $S_{p}$ should be merged. Furthermore, variable $m^{p, p+1}$ is a $N_{p} \times N_{p+1}$ binary matrix whose entries $m_{i j}^{p, p+1}$ indicate whether segment $i \in S_{p}$ and segment $j \in S_{p+1}$ are likely to be part of the same object from the original image.

Thus, for every pair of consecutive label maps, we can identify two objectives: minimizing the total cost of merging within each label-map $S_{p}$, and maximizing the total reward for agreement across label map $S_{p}$ and label map $S_{p+1}$. Fig. 2(f) illustrates this concept. Next, we discuss the objective function and the constraints associated with this program, which verify that the solutions for the three sub-problems are in agreement with each other.

The objective function of the proposed optimization program is represented by equation (11). The first summation indicates the total cost of merging within each label-map. The second summation represents the reward for agreement (merging/connecting) across two consecutive label-maps. Note that minimizing the negative total reward is equivalent to maximizing the positive total reward. The real valued parameter $\lambda$ is used to balance the total cost and total reward, and bias the final result towards more or less merging.

We now introduce the constraints needed to ensure the validity of the resulting segmentations. The range constraint (2) specifies that this is a binary program, for which the decision variables can only take 0,1 values. The connectivity constraint (3) does not permit the merging of segments which are not neighbors, within each label-map $m^{p, p}$, or across the two consecutive label-maps $m^{p, p+1}$. Constraint (4) handles symmetry: if segment $i$ is merged with segment $j$, this implies that segment $j$ is merged with segment $i$ as well. Note that there is no such constraint for the $m^{p, p+1}$ variables, which are neither square, nor symmetric 
matrices. Next, equation (15) introduces the self-merger constraint, which implies that every segment is merged with itself. This forces the diagonals of the resulting matrices $m^{p, p}$ to be 1 . Again, note that this constraint does not hold for the $m^{p, p+1}$ variables.

minimize:

$$
f=\sum_{p=1}^{N} \sum_{i=1}^{N_{p}} \sum_{j=1}^{N_{p}} m_{i j}^{p, p} C_{i j}^{p}-\lambda \sum_{p=1}^{N-1} \sum_{i=1}^{N_{p}} \sum_{j=1}^{N_{p+1}} m_{i j}^{p, p+1} R_{i j}^{p, p+1}
$$

subject to:

- Range: $(\forall i, j, p)$

- Connectivity: $(\forall i, j, p)$

$$
\begin{gathered}
m_{i j}^{p, p}, m_{i j}^{p, p+1} \in\{0,1\} \\
m_{i j}^{p, p} \leq E_{i j}^{p, p} \quad m_{i j}^{p, p+1} \leq E_{i j}^{p, p+1} \\
m_{i j}^{p, p}=m_{j i}^{p, p}
\end{gathered}
$$

- Symmetry: $(\forall i, j, p)$

- Self-merge: $(\forall i, p)$

- Transitivity: $(\forall i, j, k, p)$

$$
m_{i j}^{p, p} \geq m_{i k}^{p, p+1} m_{j k}^{p, p+1} E_{i j}^{p, p} E_{i k}^{p, p+1} E_{j k}^{p, p+1}
$$

The constraints thus far ensure the validity of the results. However the two sub-problems are still independent of each other: the minimization of the total cost within the label-maps $S_{p}$ is only affected by the cost parameters, and the maximization of the reward across two consecutive label-maps is only affected by the reward parameters. Thus, the optimization program, with the constraints thus far, will result in the trivial solution: merge nothing within each label-map $S_{p}$, merge everything across consecutive label-maps.

Therefore, we need to ensure that the two sub-problems match each other, meaning that the decision to merge segments in one label map will affect the result of the other label maps. We introduce the following transitivity constraints. As explained in Fig. 2(a), (6) implies that if segments $i$ and $j$ from label-map $S_{p}$ are both merged with segment $k$ from label-map $S_{p+1}$, then $i$ and $j$ must also be merged within $S_{p}$. This ensures that $m^{p}$ and $m^{p+1}$ are in agreement, through $m^{p, p+1}$. Note that we only enforce this constraint for neighboring segments: $E_{i j}^{p, p}$, $E_{i k}^{p, p+1}$, and $E_{j k}^{p, p+1}$ must be 1 for the constraint to be active. This is because, for situations like Fig. 2(e), the connectivity constraint from (3) will not permit the merging of two segments which do not share a pixel, leading to an infeasible program. Similarly, we consider the situations described in Fig. 2(b), 2(c) and $2(\mathrm{~d})$. Note that transitivity constraints for three nodes pertaining to the same label-map, $i, j, k \in S_{p}$ are implicit.

The number of constraints is of order $O\left(n^{3}\right)$ in the total number of segments, due to the transitivity constraints. Since the transitivity constraints are only active for neighboring segments, the size of the problem can be bounded by $O\left(n^{2}\right)$ as in [14].

We have thus formulated a Binary Integer Program (BIP) which simultaneously corrects $N$ over-segmentations of an image, while maximizing the agreement between the resulting segmentations. However, this program is an NP-hard problem and cannot be solved in polynomial time. In the following sub-section we describe its relaxation to a Linear Program (LP). 


\subsection{Linear Relaxation}

We note that the constraints of (3), (4) and (5D) are linear. However the transitivity constraints (6) (and symmetric equivalents) are not linear, and the range constraint of (2) does not form a convex set.

We linearize the transitivity constraints as in (77). The first step results from De Morgan's law, and the second step results from negating and reordering the variables. The remaining three transitivity constraints are linearized similarly.

$$
\begin{aligned}
& m_{i j}^{p, p} \geq m_{i k}^{p, p+1} m_{j k}^{p, p+1} \cdot E_{i j}^{p, p} E_{i k}^{p, p+1} E_{j k}^{p, p+1} \\
&=\left(1-\left(\overline{m_{i k}^{p, p+1}}+\overline{m_{j k}^{p, p+1}}\right)\right) \cdot E_{i j}^{p, p} E_{i k}^{p, p+1} E_{j k}^{p, p+1} \\
&=\left(m_{i k}^{p, p+1}+m_{j k}^{p, p+1}-1\right) \cdot E_{i j}^{p, p} E_{i k}^{p, p+1} E_{j k}^{p, p+1}
\end{aligned}
$$

Further, we relax the values of the variables $m^{p, p}$, and $m^{p, p+1}$, defined in the range constraint of (2), from $\{0,1\}$ to $[0,1]$. Occasionally $m^{p, p}$, and $m^{p, p+1}$ may result in fractional values, for which we consider the values above a threshold (0.5). This converts the feasibility set into a convex set, relaxing the BIP to an LP, whose global solution is an approximation of the original BIP.

\section{$3 \quad$ Experimental Results}

We evaluate the performance of our method on a 3-D confocal volume, as well as analyze its stability using a 2-D example. The comparison metric is the $F$ measure, which is a volume based error metric and is defined as: $F=\frac{2 \mathcal{P} \mathcal{R}}{\mathcal{P}+\mathcal{R}}$, where $\mathcal{P}$ and $\mathcal{R}$ are the precision and the recall for a given ground truth volume.

Performance Evaluation: Numerical results are reported on the forty cylindrical cells of the notochord tissue in a 3-D dataset for which manually traced ground truth is available. Two over-segmentations are used for this experiment: (1) the Watershed method [6] applied to the image data, and (2) Watershed applied to the filtered image (3-D mean-filter). To solve the optimization program we used CVX, a package for specifying and solving convex programs 514.

We construct a cost function which compares the intensity histograms on the border of two neighboring segments, to that of the interior of the segments. This is given by $C_{i j}^{p}=\max \left(0, \overline{\mathcal{B}}_{i j}^{p}-\overline{\mathcal{S}}_{i j}^{p}\right)$, where $\overline{\mathcal{B}}_{i j}^{p}$ and $\overline{\mathcal{S}}_{i j}^{p}$ represent the mean intensity on the border and the interior, respectively, of two neighboring segments $i, j \in S_{p}$. A large positive value indicates the presence of a membrane, while a low value suggests a spurious boundary. The reward function for two segments $i \in S_{p}$ and $j \in S_{p+1}$ is the percentage area of overlap, expressed as $\max \left(\frac{\mathcal{A}_{i j}^{p, p+1}}{\mathcal{A}_{i}^{p}}, \frac{\mathcal{A}_{i j}^{p, p+1}}{\mathcal{A}_{j}^{p+1}}\right)$.

We compare our work to the following methods relevant to confocal membrane image segmentation. The Subjective Surface variant in [13] is a level-set method specifically designed for membrane image segmentation. It requires initialization with a seed point in every cell. The method in [2] also corrects an over-segmentation by merging segments, however this method requires training 

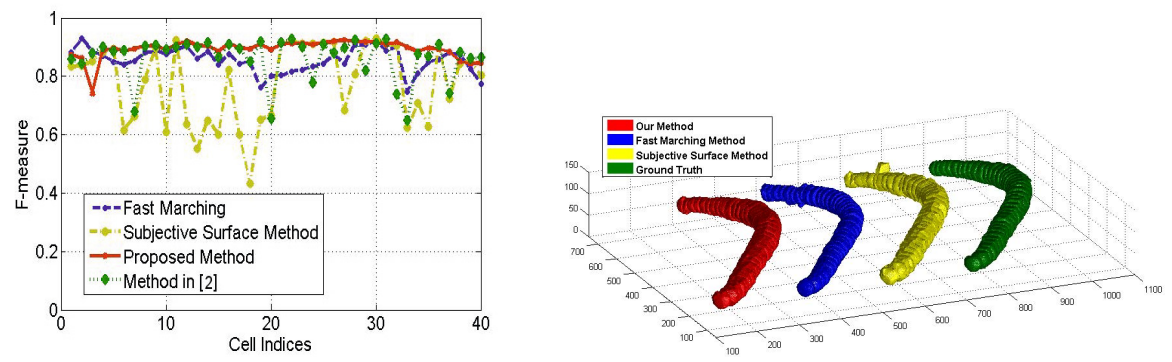

Fig. 3. Left: F-measure per notochord cell. Right: 3-D rendering of the segmented cells

Table 1. F-measure statistics on forty notochord cells in 3-D

\begin{tabular}{|c|c|c|c|}
\hline Method & Avgerage & Median & Standard Dev. \\
\hline \hline Proposed & $\mathbf{8 9 . 3 6 \%}$ & $\mathbf{9 0 . 1 3 \%}$ & $\mathbf{3 . 1 9 \%}$ \\
\hline Method in [2] & $86.25 \%$ & $88.91 \%$ & $7.30 \%$ \\
\hline Fast Marching [1] & $85.58 \%$ & $85.95 \%$ & $4.18 \%$ \\
\hline Subj. Surf. [13] & $77.87 \%$ & $82.78 \%$ & $13.63 \%$ \\
\hline Over-Segmentation \#1 & $80.01 \%$ & $81.02 \%$ & $6.37 \%$ \\
\hline Over-Segmentation \#2 & $61.62 \%$ & $64.18 \%$ & $8.73 \%$ \\
\hline
\end{tabular}

data. Lastly, the Fast Marching Method [1] computes geodesic distances in the discrete image domain from a seed-point in every cell.

As seen in Fig. 3 and Table 1, the proposed algorithm started out with two over-segmentations with $F$-scores of $80.01 \%$ and $61.62 \%$. By combining them, it achieves a score of $89.36 \%$ and outperforms the manually initialized methods in 1 and 13, and the trained method in [2, which often leak through broken boundaries and get attached to spurious edges. The lack of manual interaction is essential when handling $3-\mathrm{D}$ data with a very large number of cells. The $3-\mathrm{D}$ rendering of the segmented notochord tissue is presented in Fig. 3.

Stability Analysis: Here we investigate the impact of the input segmentations on the performance of the method. In order to evaluate this, three oversegmentations of the confocal section in Fig. 1(b) are obtained using randomly
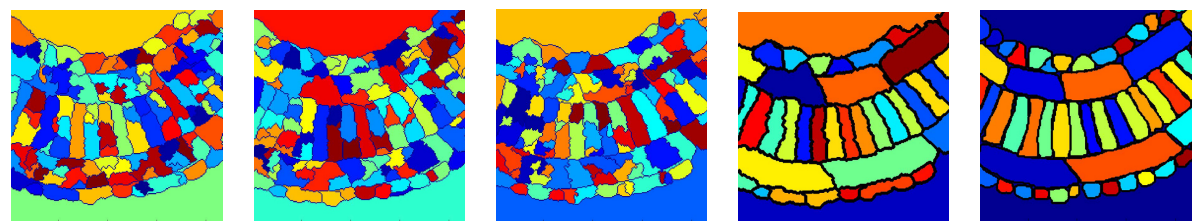

Fig. 4. Segmentation of the confocal section in Fig. 1(b) From left to right: Three randomly initialized watershed over-segmentations. Joint correction using proposed method. Ground truth segmentation. 
seeded Watershed. The proposed method is used to simultaneously correct these three over-segmentations, as shown in Fig. 4. In an unsupervised manner, the optimization framework correctly segments out the cells from the notochord and muscle tissues, which are relevant in the study of morphogenesis. The $F$-measure is computed and this process is repeated twenty times. The observed scores average to $80.32 \%$ and the standard deviation is $3.12 \%$. This indicates that the method is robust to randomized input segmentations. Furthermore, the final values of the approximately 100,000 decision variables involved in this experiment are all binary. This indicates that the LP relaxation is tight and the global solution is in fact the solution to the original BIP from section 2.1, The solver took $4 \mathrm{~s}$ on a graph of approx. 700 nodes / 7000 edges on a single core i5 at $2.5 \mathrm{GHz}$.

Conclusion: We addressed the problem of cell segmentation for 3-D confocal microscopy volumes of the Ciona. We introduced an unsupervised method that combines two or more over-segmentations in a linear optimization framework.

\section{References}

1. Cohen, L., et al.: Fast marching the global min. of active contours. In: ICIP 1996 (1996)

2. Delibaltov, D., et al.: An automatic feature based model for cell segmentation from confocal microscopy volumes. In: IEEE ISBI (2011)

3. Delibaltov, D., et al.: Robust biological image sequence analysis using graph based approaches. In: Asilomar Conference on Signals, Systems and Computers (2012)

4. Grant, C.M., Boyd, S.P.: Graph implementations for nonsmooth convex programs. In: Recent Advances in Learning and Control. LNCIS, vol. 371, pp. 95-110. Springer, Heidelberg (2008)

5. Grant, M., et al.: CVX: Matlab software for disciplined convex (2011), http://cvxr.com/cvx

6. Meyer, F., et al.: Morphological segmentation. Journal of Visual Communication and Image Representation (1990)

7. Sabuncu, M.R., et al.: A generative model for image segmentation based on label fusion. IEEE Trans. on Medical Imaging (2010)

8. Chen, T., Vemuri, B.C., Rangarajan, A., Eisenschenk, S.J.: Mixture of segmenters with discriminative spatial regularization and sparse weight selection. In: Fichtinger, G., Martel, A., Peters, T. (eds.) MICCAI 2011, Part III. LNCS, vol. 6893, pp. 595-602. Springer, Heidelberg (2011)

9. Veeman, M., et al.: Chongmague reveals an essential role for laminin-mediated boundary formation in chordate convergence and extension movements. Development Genes and Evolution (2008)

10. Veeman, M.T., et al.: Whole-organ cell shape analysis reveals the developmental basis of ascidian notochord taper. Developmental Biology (2013)

11. Warfield, et al.: Simultaneous truth and performance level estimation (STAPLE): an algorithm for the validation of image segmentation. IEEE TMI (2004)

12. Artaechevarria, X., et al.: Combination strategies in multi-atlas image segmentation: Application to brain MR data. IEEE Trans. on Med. Img. (2009)

13. Zanella, C., et al.: Cells segmentation from 3-D confocal images Of early zebrafish embryogenesis. In: IEEE TIP 2010 (2010)

14. Vitaladevuni, S.N., Basri, R.: Co-clustering of image segments using convex optimization applied to EM neuronal reconstruction. In: CVPR 2010 (2010) 